\title{
Compactons and Chaos in Strongly Nonlinear Lattices
}

\author{
Karsten Ahnert and Arkady Pikovsky \\ Department of Physics and Astronomy, \\ Potsdam University, 14476 Potsdam, Germany
}

(Dated: October 24, 2018)

\begin{abstract}
We study localized traveling waves and chaotic states in strongly nonlinear one-dimensional Hamiltonian lattices. We show that the solitary waves are super-exponentially localized, and present an accurate numerical method allowing to find them for an arbitrary nonlinearity index. Compactons evolve from rather general initially localized perturbations and collide nearly elastically, nevertheless on a long time scale for finite lattices an extensive chaotic state is generally observed. Because of the system's scaling, these dynamical properties are valid for any energy.

PACS numbers: 05.45.Yv, 63.20.Ry, 05.45.-a

Keywords: Nonlinear Hamiltonian lattices; Compactons; Space-time chaos
\end{abstract}




\section{INTRODUCTION}

Hamiltonian lattices are one of the simplest objects in nonlinear physics, nevertheless they still elude full understanding. Already the first attempt to understand nonlinear effects ended with the Fermi-Pasta-Ulam puzzle, which is still not fully resolved (see e.g. the focus issue on "The "Fermi-Pasta-Ulam" problem - the first 50 years" in [1]); another remarkable feature

found only recently is the existence of localized breathers [2]. Quite often nonlinear effects in lattices can be treated perturbatively, leading to well-established concepts of phonon interaction and weak turbulence. Beyond a perturbative account of a weak nonlinearity one is encountered with genuine nonlinear phenomena, like solitons and chaos. The level of nonlinearity usually grows with the energy, allowing one to follow a transition from linear to nonlinear regimes by pumping more energy in the lattice.

In this paper we study strongly nonlinear Hamiltonian lattices that do not possess linear terms. We restrict our attention to the simplest one-dimensional case where particles interact nonlinearly and no on-site potential is present. We choose the interaction potential in the simplest power form, thus the lattice is characterized by a single parameter - the nonlinearity index. The equations of motion obey scaling, what means that the dynamical properties are the same for all energies - only the time scale changes. Lattices of this type attracted large attention recently, in particular due to a prominent example - the Hertz lattice that describes elastically interacting hard balls, it has the nonlinearity index $3 / 2$ [3, 4, 5, 6]. We focus our study on the interplay of solitary waves and chaos in such lattices. Some 25 years ago Nesterenko [3, 7, 8, 9] has described a compact traveling wave solution in the Hertz lattice, which can be understood as a compacton. Compactons have been introduced, in a mathematically rigor form, by Rosenau and coworkers [10, 11] for a class of nonlinear PDEs with nonlinear dispersion. Compactons can be analytically found if one approximates the lattice equations with nonlinear PDEs, but less is known on the genuine lattice solutions. Below, in Section IIIB we present a numerical procedure for determining traveling waves for an arbitrary nonlinearity index, and compare these solutions with those of the approximated PDEs (Section IIIA). Furthermore, we show that compactons naturally appear from localized initial perturbations and relatively robustly survive collisions, but nevertheless evolve to chaos on a long time scale in finite lattices (Section IV). Properties of chaos are studied in Section V. We demonstrate extensivity of the chaotic state by calculating the Lyapunov 
spectrum, and study the dependence of the Lyapunov exponents on the nonlinearity index. Some open questions are discussed in the concluding Section VI.

\section{THE MODEL}

Our basic model is a family of lattice Hamiltonian systems

$$
H=\sum_{k} \frac{p_{k}^{2}}{2}+\frac{1}{n+1}\left|q_{k+1}-q_{k}\right|^{n+1},
$$

which are parameterized by one real parameter - the nonlinearity index $n$. Below we assume that $n \geq 1$. The case $n=1$ corresponds to a linear lattice. Another interesting case is $n=3 / 2$. Such a nonlinearity appears, according to the Hertz law, at the compression in a chain of elastic hard balls. For the realistic system of balls, however, the potential has the form like in (11) only for $q_{k+1}-q_{k}<0$, for $q_{k+1}-q_{k}>0$ no attracting force is acting. A simplified realization of such a system is the toy "Newton's cradle", which possesses the same Hertzian interaction law. However the standard "Newton's cradle" consists of a few balls (typically 5), which are not enough for the formation of stationary traveling waves. Furthermore, slight intervals between adjacent beads are not excluded, contrary to experiments [4, 7] where great care is taken to let the beads in effective contact. For different aspects of the Hertz chain see refs. [6, 9, 12, 13, 14, 15, 16, 17, 18, 19, 20, 21], a review article [22], and references therein. Contrary to this, in our model (11) we assume both repulsive and attracting forces.

Note that the potential in (1) is generally non-smooth, except for cases $n=1,3,5, \ldots$. Although the dynamics can be easily studied in non-smooth situations as well, we will mainly focus below on the simplest smooth non-trivial case $n=3$.

The lattice equation of motion read

$$
\ddot{q}_{k}=\left|q_{k+1}-q_{k}\right|^{n} \operatorname{sign}\left(q_{k+1}-q_{k}\right)-\left|q_{k}-q_{k-1}\right|^{n} \operatorname{sign}\left(q_{k}-q_{k-1}\right) .
$$

Since on the right hand side of (2) only differences enter, it is convenient to introduce the difference coordinates $Q_{k}=q_{k+1}-q_{k}$. Then the equations of motion are transformed to

$$
\ddot{Q}_{k}=\left|Q_{k+1}\right|^{n} \operatorname{sign}\left(Q_{k+1}\right)-2\left|Q_{k}\right|^{n} \operatorname{sign}\left(Q_{k}\right)+\left|Q_{k-1}\right|^{n} \operatorname{sign}\left(Q_{k-1}\right) \text {. }
$$

Note, that a solitary wave in the variables $Q_{k}$ corresponds to a kink (shock-like wave) in the variables $q_{k}$. 
Conservation laws. The equations of motion possess two conservation laws: the energy and the total momentum, the latter can be trivially set to zero by transforming into a moving reference frame.

Scaling. As mentioned in Refs. [3, 5, 9], the lattice (1) has remarkable scaling properties, due to homogeneity of the interaction energy. It is easy to check, that the Hamiltonian can be rescaled according to

$$
q=a \tilde{q}, \quad p=a^{\frac{n+1}{2}} \tilde{p}, \quad H=a^{n+1} \tilde{H}, \quad t=a^{\frac{1-n}{2}} \tilde{t} .
$$

Note, that this scaling involves only the amplitude and the characteristic time of the solutions: by decreasing the amplitude one obtains new solutions having the same spatial structure but evolving slower. We will see that this property has direct consequences for the properties of traveling waves and of chaos.

\section{TRAVELING SOLITARY WAVES}

In this section localized traveling waves are investigated, first in a quasicontinuous approximation ( $\mathrm{QCA}$ ) and then via numerical solution of the lattice equations. A mathematically rigor proof of the existence of solitary waves in Hamiltonian lattices of type (1) has been given in Refs. [23, 24].

\section{A. Quasicontinuous approximation}

Here, we represent the solution of the lattice equations (3) as a function of two continuous variables $Q(x, t)$. We are seeking for solitary waves which do not change their sign, for definiteness we consider $Q \geq 0$ (this consideration is therefore suitable for lattices where the nonlinearity index is different for positive and negative displacements $Q$, e.g. for the Hertz lattice of elastic balls). We present two approaches to find a continuous version of the lattice. In the first one, we approximate the differences between two displacements $Q$, while in the second one the displacement $q$ at each lattice site is expanded directly. 


\section{Expansion of differences}

Here we look for a direct quasicontinuous approximation of Eq. (3). Expanding the difference coordinates $Q_{k}$ up to the fourth order we obtain

$$
Q_{k \pm 1}^{n} \approx Q^{n}(x, t) \pm h\left[Q^{n}(x, t)\right]_{x}+\frac{h^{2}}{2}\left[Q^{n}(x, t)\right]_{x x} \pm \frac{h^{3}}{6}\left[Q^{n}(x, t)\right]_{x x x}+\frac{h^{4}}{24}\left[Q^{n}(x, t)\right]_{x x x x}
$$

where $h$ is the spatial difference between two lattice sites and the subscripts denote differentiation with respect to $x$. Inserting (5) into (3) and setting $h=1$ one arrives at the partial differential equation

$$
[Q(x, t)]_{t t}=\left[Q^{n}(x, t)\right]_{x x}+\frac{1}{12}\left[Q^{n}(x, t)\right]_{x x x x}
$$

Equation (6) belongs to a class of strongly nonlinear PDEs, because the dispersion term with

the fourth derivative is nonlinear. The equation does not possess linear wave solutions (this situation has been called "sonic vacuum" by V. Nesterenko), but it has nontrivial nonlinear ones. In this way it is very similar to a family of strongly nonlinear generalizations of the Korteveg-de Vries equation, studied in [10] and can be considered as a strongly nonlinear version of the Boussinesq equation [11].

Now we seek for traveling wave solutions of (6), by virtue of the ansatz

$$
Q(x, t)=Q(x-\lambda t)=Q(s)
$$

Then (7) reduces to the ODE

$$
\lambda^{2} Q_{s s}=\left[Q^{n}\right]_{s s}+\frac{1}{12}\left[Q^{n}\right]_{s s s s} .
$$

Furthermore, we assume that the solution tends to zero as $s \rightarrow \pm \infty$, thus after integrating twice we obtain

$$
\lambda^{2} Q=Q^{n}+\frac{1}{12}\left[Q^{n}\right]_{s s} .
$$

This equation also appears in the traveling waves ansatz for the $K(n, n)$-equation in [10]. Eq. (9) can be solved for an arbitrary power $n$ by

$$
Q(s)=|\lambda|^{m} A_{1} \cos ^{m}\left(B_{1} s\right)
$$

with

$$
m=\frac{2}{n-1}, \quad A_{1}=\left(\frac{n+1}{2 n}\right)^{\frac{1}{1-n}}, \quad B_{1}=\sqrt{3} \frac{n-1}{n} .
$$




\section{Expansion of displacements}

Another type of quasi-continuum can be obtained if we approximate Eq. (2). Now the displacement $q$ at each lattice site is written as a continuous variable, what for the same order of the spatial derivative as in (5) gives:

$$
q_{k \pm 1}=q \pm h q_{x}+\frac{h^{2}}{2} q_{x x} \pm \frac{h^{3}}{6} q_{x x x}+\frac{h^{4}}{24} q_{x x x x}
$$

Inserting this expansion into the equations of motion (2) and collecting all terms up to order of $h^{n+3}$ yields

$$
[q]_{t t}=h^{n+1}\left[q_{x}^{n}\right]_{x}+\frac{h^{n+3}}{12}\left(\left[q_{x}^{n}\right]_{x x x}-\frac{n(n-1)}{2}\left[q_{x}^{n-2} q_{x x}^{2}\right]_{x}\right) .
$$

This equation is the long wave approximation of Nesterenko [3, 9]. To compare it with Eq. (6) , we differentiate (12) with respect to $x$, define $\tilde{Q}=h q_{x}$ and set $h=1$ :

$$
[\tilde{Q}]_{t t}=\left[\tilde{Q}^{n}\right]_{x x}+\frac{1}{12}\left(\left[\tilde{Q}^{n}\right]_{x x x x}-\frac{n(n-1)}{2}\left[\tilde{Q}^{n-2} \tilde{Q}_{x}^{2}\right]_{x x}\right) .
$$

One can see that there is an additional term in (13) compared to (66). This is not so much surprising, as these two quasi-continuous approximations correspond to expansions at the different positions of the original lattice, this effect is well-known for approximations of Hamiltonian lattices with PDEs [25]. Because in the problem we do not have a small parameter (the lattice spacing $h=1$ is not small compared to the wave length), none of the equations (6),(13) can be expected to be exact in some asymptotic sense. Instead, one has to justify them by comparing the solutions with those of the full lattice problem, see Section IIIB below.

To find traveling waves in the direct expansion we use again the ansatz (7) $\tilde{Q}(x, t)=$ $\tilde{Q}(x-\lambda t)=\tilde{Q}(s)$. Inserting this ansatz and integrating twice yields then an analogon to (9)

$$
\lambda^{2} \tilde{Q}=\tilde{Q}^{n}+\frac{1}{12}\left[\tilde{Q}^{n}\right]_{s s}-\frac{n(n-1)}{24} \tilde{Q}^{n-2} \tilde{Q}_{s}^{2}
$$

One partial solution of this ordinary differential equation can also be written as

$$
\tilde{Q}(s)=|\lambda|^{m} A_{2} \cos ^{m}\left(B_{2} s\right)
$$

but with different constants $A_{2}$ and $B_{2}$ (cf. [19, 26])

$$
m=\frac{2}{n-1}, \quad A_{2}=\left(\frac{2}{1+n}\right)^{\frac{1}{1-n}}, \quad B_{2}=\sqrt{6 \frac{(n-1)^{2}}{n(n+1)}} .
$$


The solutions (10) and (15) do not satisfy boundary conditions, moreover, they intersect with another, trivial solution of (8) $Q=0$. Remarkably, because of the degeneracy of Eq. (8) and Eq. (14) at zero, one can merge periodic solutions (10) and (15) with the trivial solution $Q=0$ (see a detailed discussion in [10, 11]):

$$
Q(s)= \begin{cases}|\lambda|^{m} A_{i} \cos ^{m}\left(B_{i} s\right) & |s|<\frac{\pi}{2 B_{i}} \\ 0 & \text { else. }\end{cases}
$$

with $i=1,2$. This gives a compacton - a solitary wave with a compact support - according to definition [10, 11]. For other, non-solitary solutions of (13), see e.g. [9, 26]. Note that due to the symmetries $x \rightarrow-x, Q \rightarrow-Q$, solitary waves with both signs of velocity $\lambda$ and of amplitude $A$ are the solutions.

It is important to check the validity of solution (16) by substituting it back to (8) or (13). Then no terms are singular for the case $m>2$ only, i.e. for $n<2$. Thus, the constructed compacton solution (16) is, strictly speaking, not valid for strong nonlinearities $n \geq 2$. This conclusion is, however, only of small relevance for the original lattice problem. Indeed, the PDE (6) or (12) is only an approximation of the lattice problem: because the spatial extent of solution (16) is finite, there is no small parameter allowing us to break expansion (5) or (11) somewhere. Just breaking it after the fourth derivative is arbitrary and can be justified only by the fact, that in this approximation one indeed finds reasonable solutions at least for some values of $n$. A real justification can come only from a comparison with the solutions of the lattice equations, to be discussed in the next subsection. And there we will see that the solution can be found both for weak and strong nonlinearities $n>2$.

\section{B. Traveling waves in the lattice}

In the lattice, the traveling wave ansatz reads $Q_{k}(t)=Q(k-\lambda t)=Q(s)$. Inserting this ansatz into the lattice equations (3) yields

$$
\lambda^{2} Q^{\prime \prime}(s)=Q^{n}(s-1)-2 Q^{n}(s)+Q^{n}(s+1) .
$$

We employ now the scaling (4) and set $\lambda=1$. As demonstrated in [27], this advanced-delay differential equation can be equivalently written as an integral equation

$$
Q(s)=\int_{s-1}^{s+1}(1-|s-\xi|) Q^{n}(\xi) \mathrm{d} \xi
$$




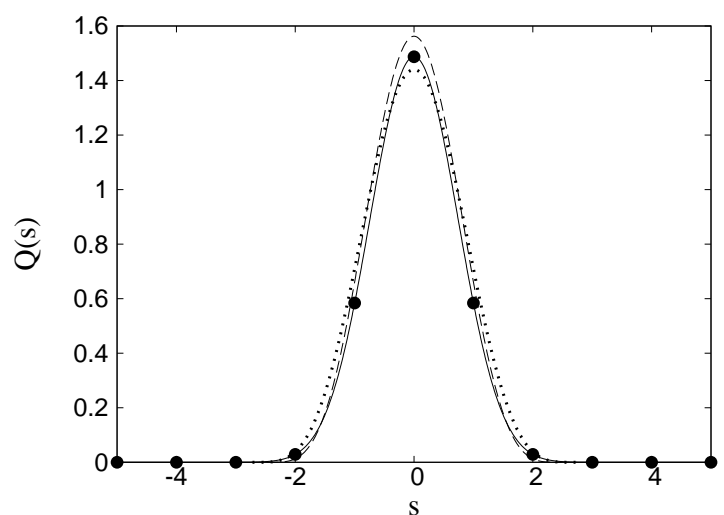

(a)

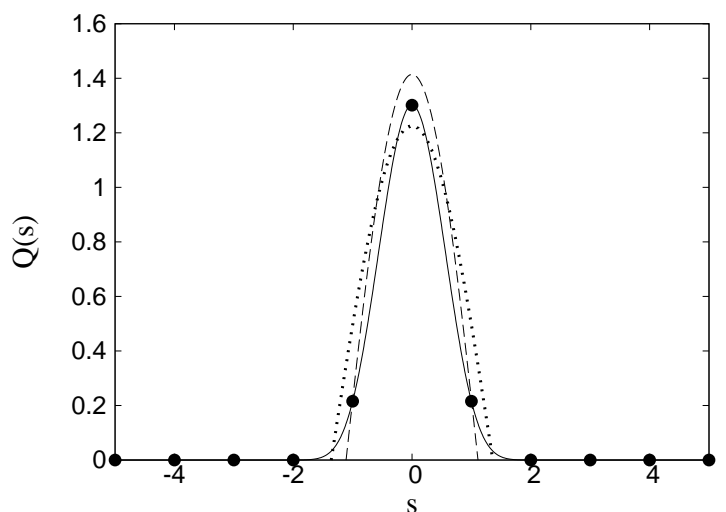

(c)

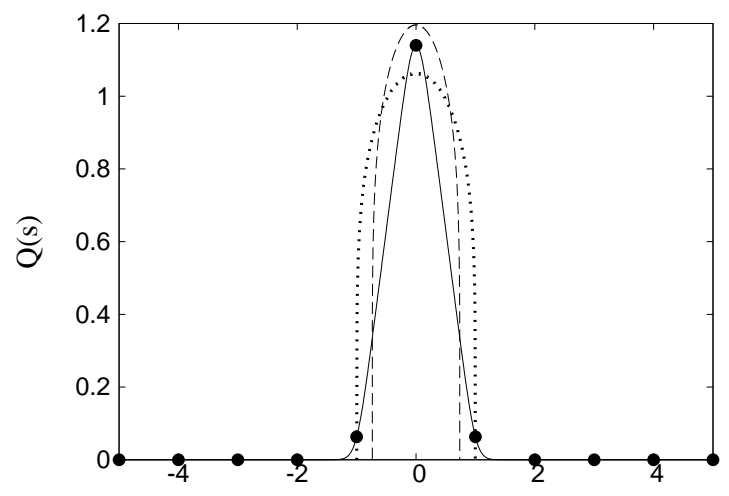

(e)

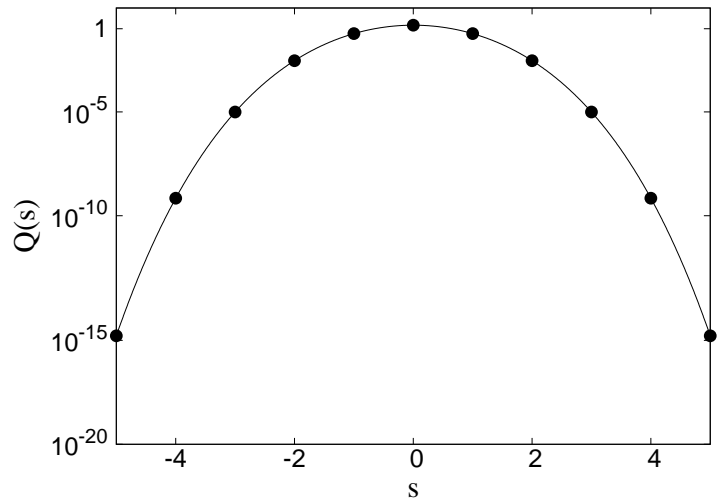

(b)

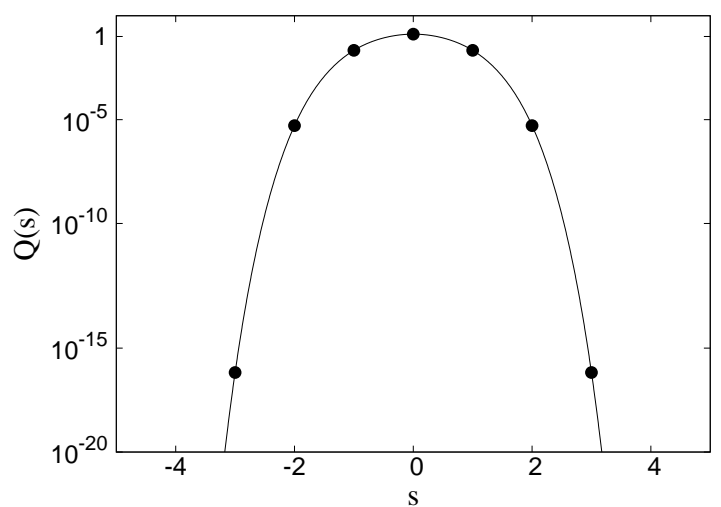

(d)

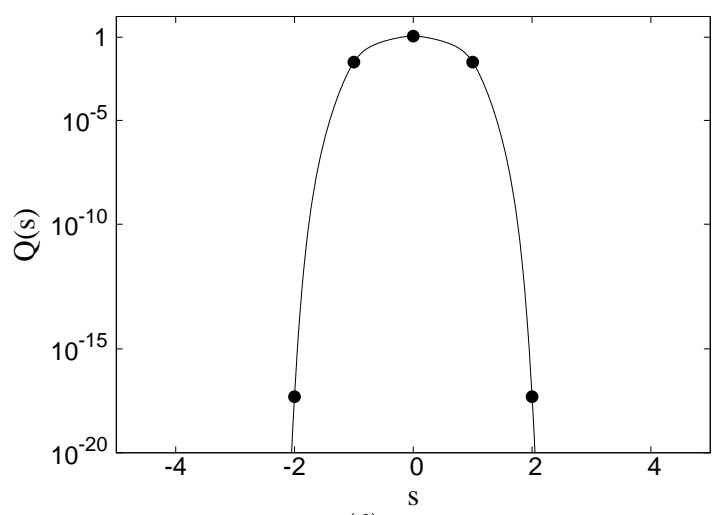

(f)

FIG. 1: The traveling waves obtained from (19) for various powers $n$. Markers show the wave on the lattice, dotted lines show the corresponding solutions of the quasi-continuous approximation (6) and dashed lines show the solutions of the QCA (13). Left column: normal scale, right column: logarithmic scale. (a,b) $n=3 / 2 ;(\mathrm{c}, \mathrm{d}) n=3,(\mathrm{e}, \mathrm{f}) n=11$. Note that the width $w$ of the compacton decreases as $n$ increases. 
[One can easily check the equivalence by differentiating (18) twice.] We can now, following the approach of V. Petviashvili [28, 29], construct an iterative numerical scheme to solve the integral equation (18). Starting with some initial guess $Q_{0}$, one constructs the next iteration via

$$
\begin{aligned}
Q_{i+1} & =\left(\frac{\left\|Q_{i}\right\|}{\left\|Q_{*}\right\|}\right)^{\alpha} Q_{*} \text { and } \\
Q_{*} & =\int_{s-1}^{s+1}(1-|s-\xi|) Q_{i}^{n}(\xi) \mathrm{d} \xi,
\end{aligned}
$$

(practically, we used the $L_{1}$-norm for $\|\cdot\|$ ). We have used $\alpha=\frac{n}{n-1}$ that ensured convergence of the iterative scheme. The integral in (19) was numerically approximated by virtue of a 4th-order Lagrangian integration scheme [30]. In Fig. 1 the traveling waves for various powers $n$ are shown. Using the logarithmic scale one clearly recognizes the compact nature of the waves.

In Fig. 2 we show the dependencies of the total energy $E$, the solution $L_{1}$-norm $N_{L_{1}}$, and the amplitude $Q_{\max }$ of the found waves on the nonlinearity index $n$, for a fixed wave velocity of $\lambda=1$. Remarkably, the effective width $N_{L_{1}} / Q_{\max }$ decreases with increasing nonlinearity index and it seems that the profile of the compacton converges to a triangular shape as $n \rightarrow \infty$. A similar result has been obtained in [20], where the dependence of the pulse velocity on the nonlinearity index has been analyzed for large $n$ in the binary collision approximation.

\section{Estimation of the tails}

It is clear from the integral form (18), that the solution cannot have a compact support. In this section we estimate the decay of the tails. We start with (18) and substitute $Q(s)=$ $e^{-f(s)}$ :

$$
Q(s)=\int_{s-1}^{s+1}(1-|s-\xi|) e^{-n f(\xi)} \mathrm{d} \xi .
$$

We consider the tail for large $s>0$, if we assume a rapid decay of $Q(s)$, then the integrand in (20) has a sharp maximum at $s-1$. Thus we can approximate the integral using the Laplace method. At the maximum we expand $f(\xi)$ into a Taylor series around $s-1$, keeping 


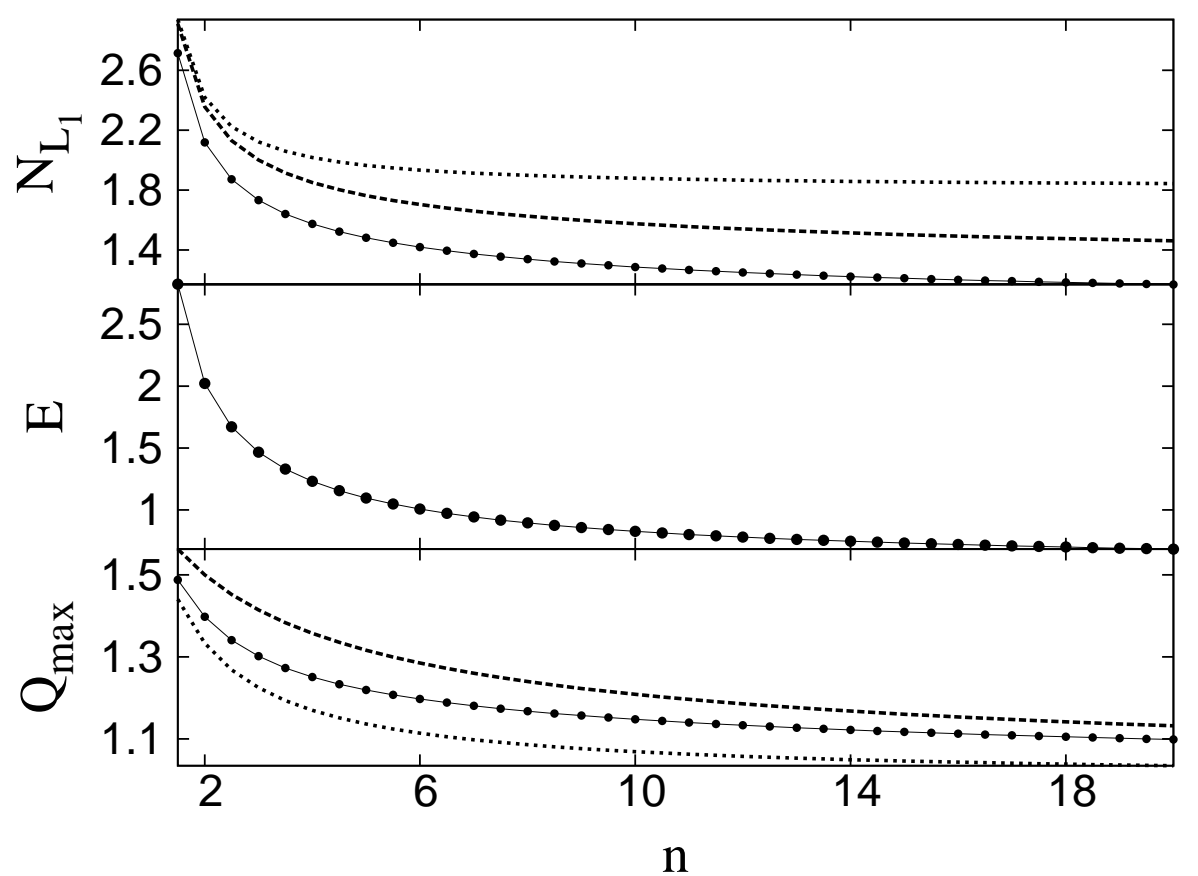

FIG. 2: The dependency of the amplitude $Q_{\max }$, the energy $E$ and the $L_{1}$-norm $N_{L_{1}}$ of a compacton on the nonlinearity index $n$. In this plots $\lambda=1$. For comparison, the curves from the quasicontinuous approximation are shown with dotted lines for the Eq. (6) and with dashed lines for $\mathrm{Eq} .(13)$.

only the leading first-order term:

$$
Q(s) \approx \int_{s-1}^{s+1}(1-|s-\xi|) \exp \left\{-n f(s-1)-n f^{\prime}(s-1)(\xi-(s-1))\right\} \mathrm{d} \xi
$$

We shift the integration range

$$
Q(s) \approx e^{-n f(s-1)} \int_{0}^{2} \xi e^{-n f^{\prime}(s-1) \xi} \mathrm{d} \xi
$$

where we also replace the decreasing part of the kernel with $\xi$. Since this integrand decreases very fast, we can set the upper bound of the integration to infinity, then by partial integration we obtain

$$
Q(s)=e^{-f(s)} \approx \frac{e^{-n f(s-1)}}{\left[n f^{\prime}(s-1)\right]^{2}} .
$$

Taking the logarithm of this equation yields

$$
-f(s)=-n f(s-1)-2 \log \left[n f^{\prime}(s-1)\right]
$$




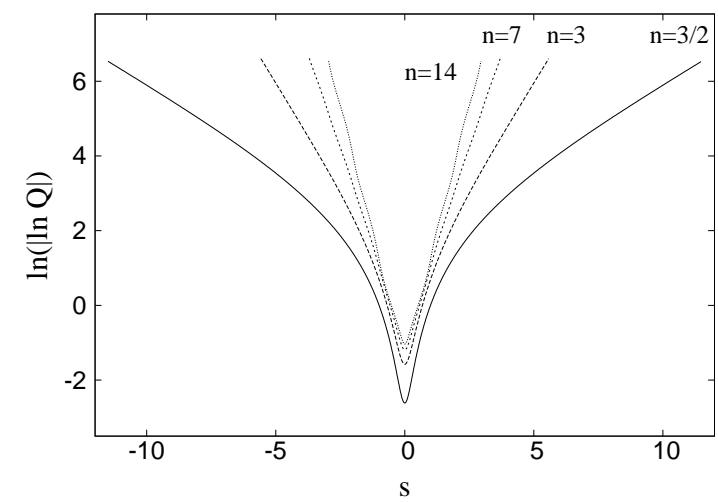

(a)

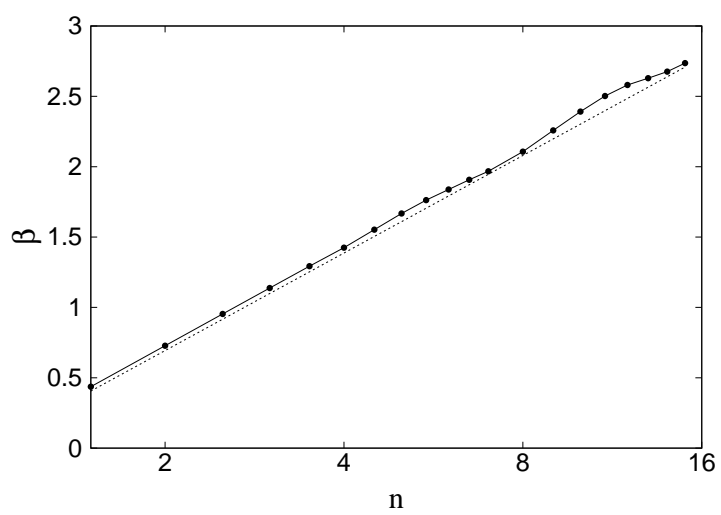

(b)

FIG. 3: (a) The tails of the compactons in double logarithmic scale. (b) Comparison of the estimate (27) with the compactons obtained from (19).

Since we expect that $f(s)$ is a rapidly growing function of $s$, we can neglect the logarithmic term and obtain

$$
f(s)=n f(s-1)
$$

This equation is solved by

$$
f(s)=C n^{s}=C e^{\log (n) s}
$$

where $C$ is an arbitrary constant. Finally we obtain that the tail decays super-exponentially:

$$
Q(s)=e^{-f(s)} \approx e^{-C n^{s}}=\exp [-C \exp (\log (n) s)]
$$

This expression was first obtained by Chatterjee [5] using a direct expansion of the advanceddelayed equation (17).

In Fig. 3(a) we show the tails of the compactons for various values of $n$ and in Fig. 3(b) we compare the estimated decay rate (27) with compactons obtained numerically from the traveling wave scheme (19). To obtain the double logarithmic decay rate $\beta=d \log (|\log (Q(s))|) / d s$, we first compute $\log (|\log (Q(s))|)$ and then the derivate is calculated using a spline smoothing scheme [31]. To suppress small oscillations of the tails we average the numerical obtained derivative in the last $1 / 6$ of the compacton domain. The numerical value of $\beta$ is shown in Fig. 3(b), Both coincide very well. 


\section{EVOLUTION AND COLLISIONS OF COMPACTONS}

\section{A. Appearance of compactons from localized initial conditions}

The compact solitary waves constructed in the previous section are of relevance only if they evolve from rather general, physically realizable initial conditions. For an experimental significance (see [4, 7] for experiments with Hertz beads) it is furthermore important, that the emerging compact waves establish on relatively short distances, otherwise dissipation (which has not been considered here) will suppress their formation. We illustrate this in Figs. 4,5. There we report on a numerical solution of the lattice equations (2) on a finite lattice of length $N=128$ (so that at the boundaries $\ddot{q}_{1}=\left|q_{2}-q_{1}\right|^{n} \operatorname{sign}\left(q_{2}-q_{1}\right)$ and $\ddot{q_{N}}=-\left|q_{N}-q_{N-1}\right|^{n} \operatorname{sign}\left(q_{N}-q_{N-1}\right)$ holds $)$. One of the quantities we report is the local energy at site $k$ defined as

$$
\mathcal{E}_{k}=\frac{p_{k}^{2}}{2}+\frac{1}{2(n+1)}\left(\left|q_{k+1}-q_{k}\right|^{n+1}+\left|q_{k}-q_{k-1}\right|^{n+1}\right)
$$

As an initial condition we have chosen a kink in the variables $q_{k}: q_{k}=(n+1)^{1 /(n+1)}$ for $k>64$ and $q_{k}=0$ elsewhere. This profile has unit energy, it corresponds to the localized initial condition in the variable $Q: Q_{k}=\delta_{k, 64} \cdot(n+1)^{1 /(n+1)}$. The evolution of different variables is shown in Fig. 4, From the initial pulse of $Q$, a series of compactons with alternating signs is emitted in both directions. The amplitude of the perturbation near the initially seeded site decreases and correspondingly increases a characteristic time of the evolution. We expect that at large times, compactons with small amplitudes will continue to detach. In Fig. 5 we show the evolution from the initial step for different nonlinearities $n=1.5,3,10$. The plots look very similar and compactons are emitted in every case. The number of the emitted compactons and their amplitudes depend on the nonlinearity index.

In our next numerical experiment we studied the emergence of compactons not from a sharp step in the coordinates $q_{k}$, but from localized random initial conditions. In Fig. 6(a) we show a typical evolution in a lattice of length $N=512$ (with nonlinearity index $n=3$ ) resulting from random initial conditions $q_{k}$ in the small region $N / 2-5 \leq k<N / 2+5$ around the center of the lattice. In this region the coordinates $q_{k}$ have been chosen as independent random numbers, identically and symmetrically uniformly distributed around zero, while $p_{k}(0)=0$. Furthermore, the energy of the lattice was set to $E=1$ by rescaling. In a particular realization of Fig. 6(a), at the initial state two compactons emerge to the right 


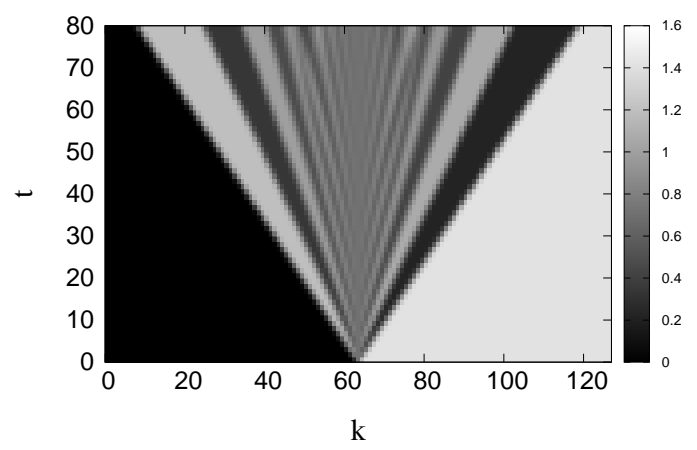

(a)

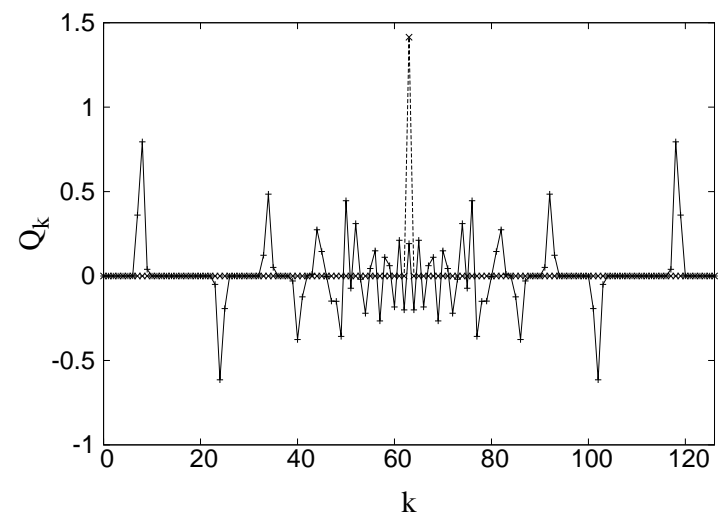

(c)

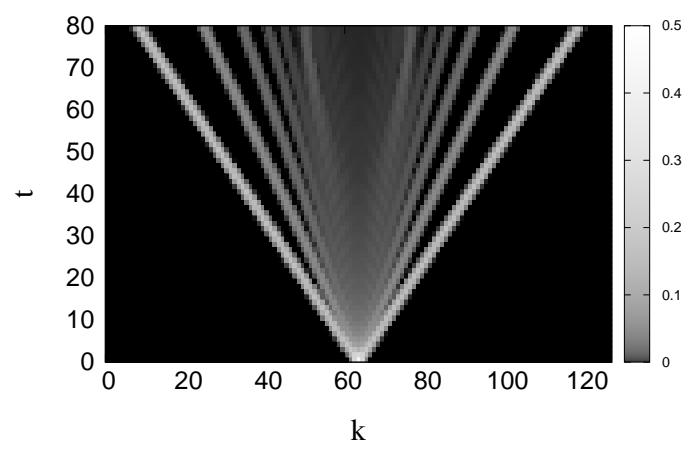

(b)

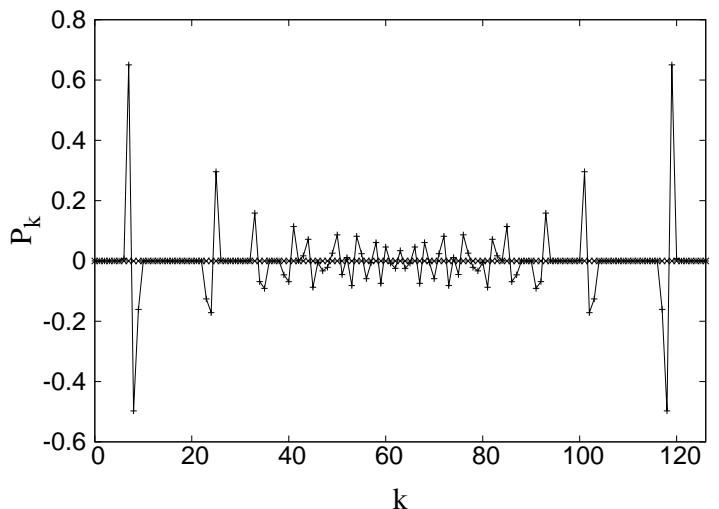

(d)

FIG. 4: Evolution from an initial step for the nonlinearity index $n=3$, the lattice length is $N=128$ and open boundary conditions (hence $\ddot{q}_{1}=\left(q_{2}-q_{1}\right)^{n}$ and $\left.\ddot{q}_{N}=-\left(q_{N}-q_{N-1}\right)^{n}\right)$ are used. The initial conditions are $q_{k}(t=0)=(n+1)^{1 /(n+1)}$ for $k>64$ and 0 else, initial momenta are zero. Different plots show different quantities of the lattice: (a) the coordinates $q_{k}$; (b) the energy $\mathcal{E}_{k}$ defined in (28); (c) the difference coordinates $Q_{k}=q_{k+1}-q_{k}$ at time $t=80$, the initial state at $t=0$ is shown here as the dashed line and $(\mathrm{d})$ the difference momenta $P_{k}=p_{k+1}-p_{k}$ at $t=80$. The compactons originating from this initial state are clearly separated near the borders of the chain, those in the middle part are still overlap-ed.

and four compactons to the left. In the center of the lattice a chaotic region establishes and slowly spreads over the lattice, possibly emitting more compactons on a longer time scale. In Fig. 6(b) we perform a statistical analysis of this setup by showing the energy distribution of compactons emitted from localized random initial conditions as described above. This distribution was obtained from 60000 simulations, in each simulation the energy of the emitted compactons have been determined and counted. The functional form of the 


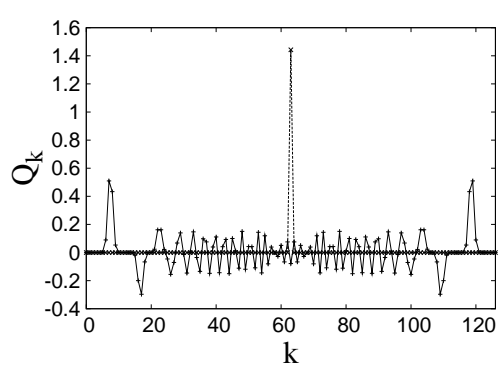

(a)

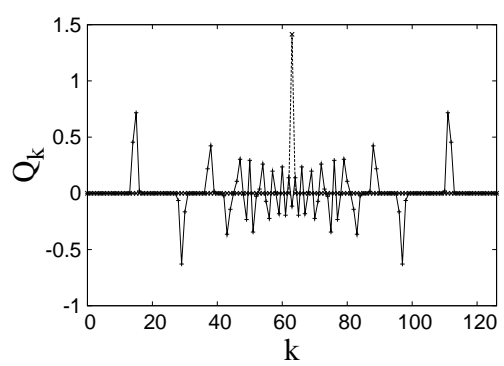

(b)

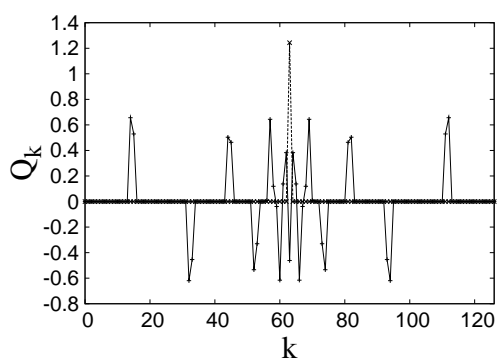

(c)

FIG. 5: Evolution of an initial step (like in Fig. 4) for various nonlinearity indices (a) $n=1.5$, (b) $n=3$ and (c) $n=10$. The initial state is shown as dashed line, the solid line is the state at time $t=80$.

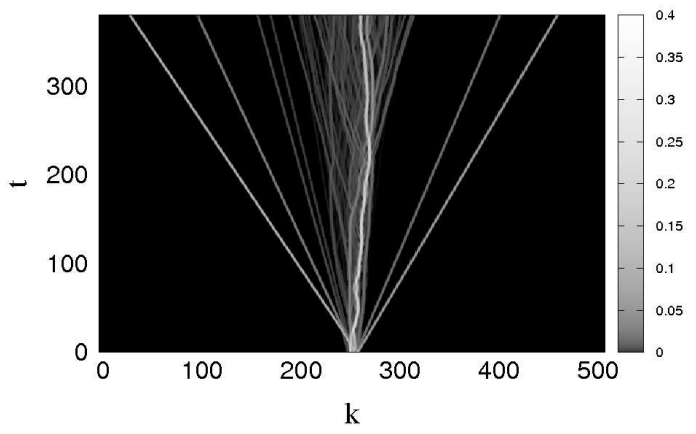

(a)

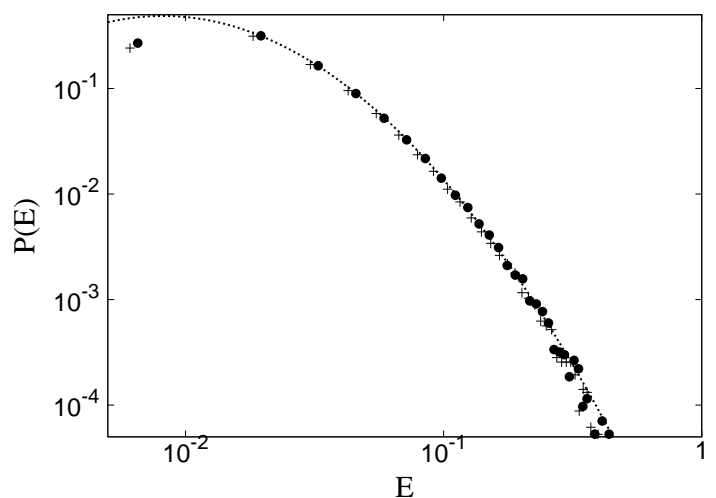

(b)

FIG. 6: (a) Compactons emerging from localized random initial conditions. The nonlinearity index is $n=3$. The gray scale corresponds to the energy (28) of the lattice site. (b) Energy distribution of the compactons emitted from localized random initial conditions. The statistics was obtained from 60000 simulations; in each simulation the lattice was integrated to the time $T=1000$ and the energy distribution of the compactons emerged to the right (black circles) and the left (crosses) have been determined. The distributions obeys in very good approximation $P(E) \sim E^{-a \log (E)-b}$, with $a=0.57$ and $b=5.47$.

distribution obeys in very good approximation $P(E) \sim E^{-a \log (E)-b}$, with $a=0.57$ and $b=5.47$. 


\section{B. Collisions of compactons}

As we have demonstrated above, compactons naturally appear from rather general initial conditions. To characterize their stability during the evolution, we study their stability to the collisions. This study is not complete but only illustrative, as in Fig. 7 we exemplify different cases of collision of two compactons in a lattice with $n=3$. These six setups present all possible scenarios of two compactons: (i) two colliding compactons with the same amplitudes, (ii) two compactons with different amplitudes moving toward each other, and (iii) two compactons with different amplitudes moving in the same direction and passing each other. Each of these three cases has two sub-cases, because the amplitudes can have the same or different sign. It should be mentioned, that these six collisions do not represent the complete picture of all collision, moreover we have not varied parameters such as the distance between two colliding compactons or their amplitudes.

In all the cases presented, the initial compactons survive the collision: they are not destroyed although they do not come out of the collision unchanged. In all cases the collision is non-elastic, some small perturbations (that presumably on a very long time scale may evolve into small-amplitude compactons) appear.

Because of this non-elasticity, on a finite lattice after multiple collisions initial compactons get destroyed and a chaotic state appears in the lattice, as illustrated in Fig. 8. There we show the evolution of the two compactons with the same amplitude and sign of the amplitude for three different nonlinearities: $n=3, n=9 / 2$ and $n=11$. In the first two cases the chaotic state establishes relatively fast. In the third simulation with $n=11$ the situation is different. Here the chaotic state does not appear even on a very long time scale. We run the simulation for very long times up to $T=2 \cdot 10^{5}$, but could not observe the development of a chaotic state. We have checked this phenomenon also for higher values of $n$ with the same result. Presumably, these initial conditions lie on a stable quasiperiodic orbit or are extremely close to a such one.

\section{CHAOS IN A FINITE LATTICE}

As demonstrated above, in a finite lattice general initial conditions evolve into a chaotic state. For characterization of chaos we use Lyapunov exponents. The chaotic state of the 


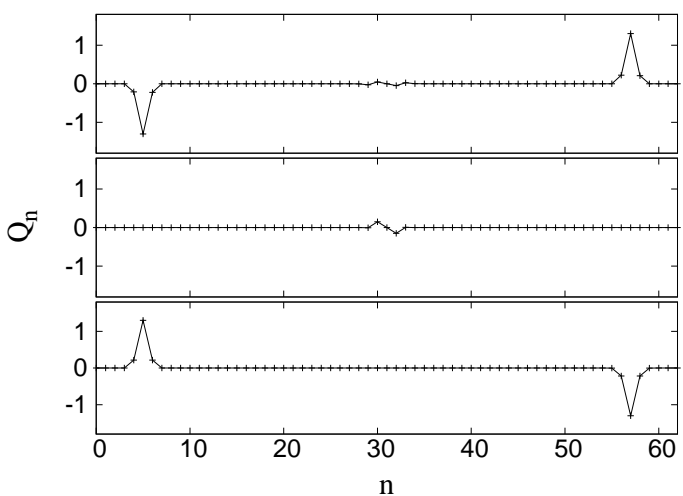

(a)

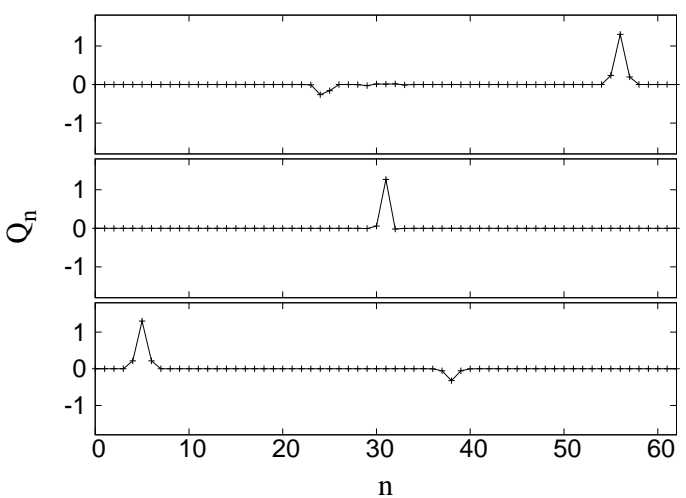

(c)

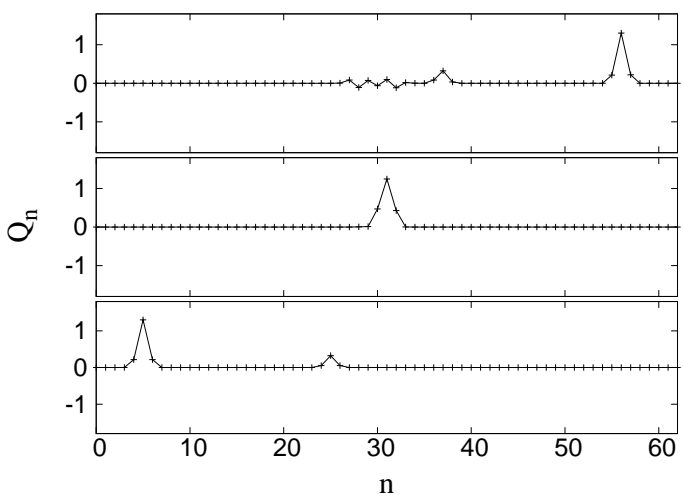

(e)

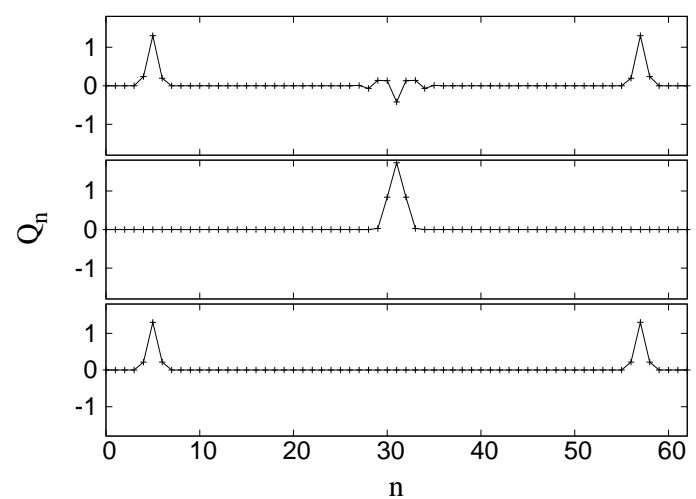

(b)

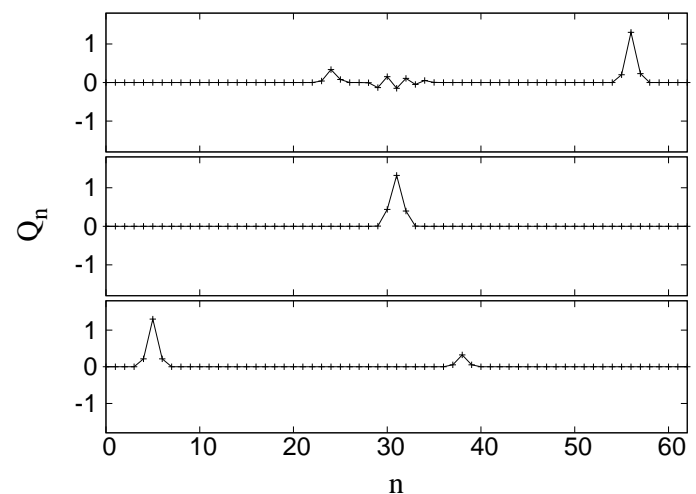

(d)

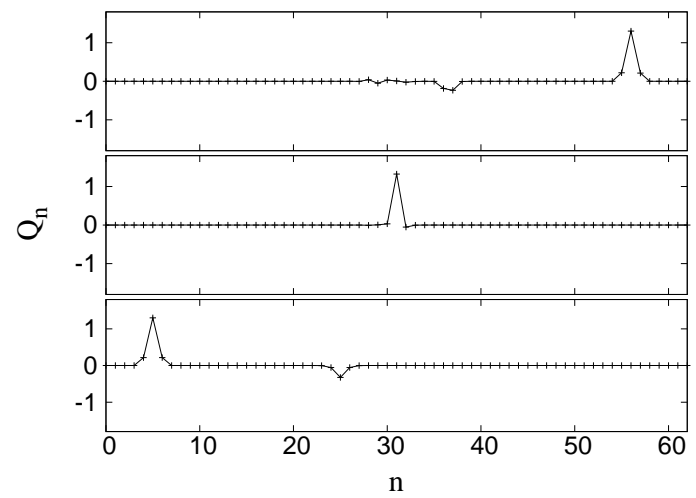

(f)

FIG. 7: Collisions of compactons in the Hamiltonian lattice with $n=3$, shown are difference coordinates $Q_{k}$. We have considered 6 different collision scenarios, in each plot the lower panel is the initial configuration of the lattice, the middle panel is the state of the lattice at some time during the maximal overlap, and the upper panel shows the lattice past the collision. (a) Compactons of equal energy having opposite amplitudes and velocities; (b) compactons of equal energies and amplitudes but opposite velocities; (c) compactons of different energies having amplitudes and velocities of opposite signs; (d) compactons of different energies having amplitudes of the same and velocities of opposite signs; (e) compactons of different energies having velocities and amplitudes of the same sign; (f) compactons of different energil $\oint_{\mathrm{g}}$ having velocities of the same sign and amplitudes of opposite signs. 


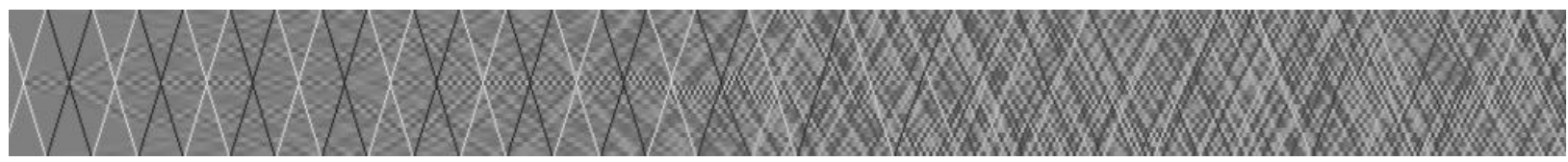

(a)

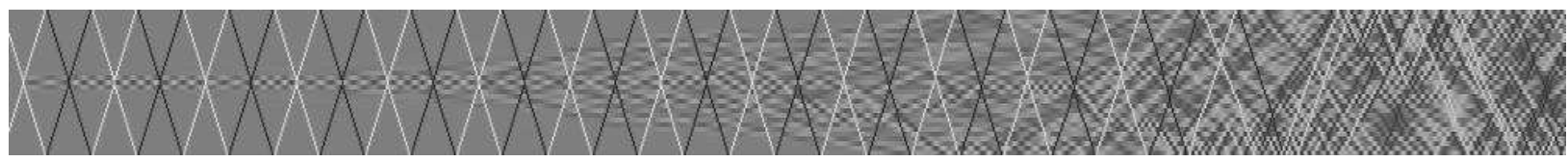

(b)

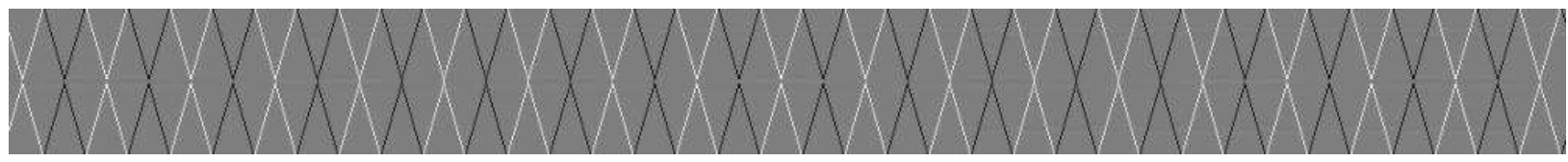

(c)

FIG. 8: Collisions of compactons and emergence of chaos after multiple collisions. Different plots show different nonlinearity indices (a) $n=3$, (b) $n=9 / 2$ and (c) $n=11$. Time increases from left to right and the difference coordinates $Q_{k}$ are shown in gray scale. Remarkably the elasticity of the collision increases with increasing nonlinearity index $n$, so that practically no irregularity appears at $n>10$.

lattice has also been characterized in [22, 32] by the means of the velocity distribution of the lattice site. It has been found that the lattice possesses a quasi-non-equilibrium phase, characterized by a Boltzmann-like velocity distribution but without energy equipartition.

First, we check that chaos in the lattice is extensive, i.e. the Lyapunov exponents form a spectrum when the system size becomes large (Fig. 9(a)). This property allows us to extend the calculations of finite lattices to the thermodynamic limit. Note, that due to the two conservation laws, four Lyapunov exponents vanish; we have not found any more vanishing exponents, indicating the absence of further hidden conserved quantities.

For a lattice of length $N=16$ the dependence of the Lyapunov exponents on the nonlinearity is shown in Fig. 9(b), For a fixed total energy (we have set $H=N=16$ in these calculations) the Lyapunov exponents grow with the nonlinearity index. The plot presented in Fig. 9(c) indicates that $\lambda_{\max } \propto \log n$, although we did not consider very high nonlinearity indices to make a definite conclusion on the asymptotics for large $n$.

We stress here that because of the scaling of the strongly nonlinear lattices under con- 


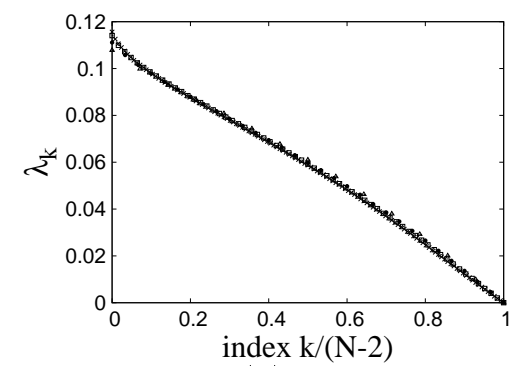

(a)

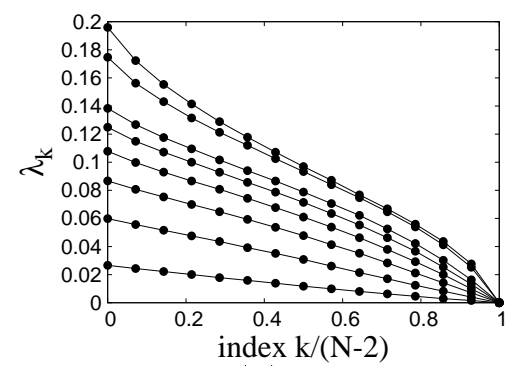

(b)

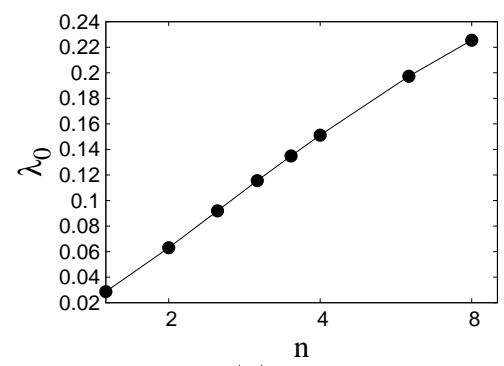

(c)

FIG. 9: Lyapunov exponents of the Hamiltonian (1). (a) The Lyapunov spectra for one fixed nonlinearity index $n=3$ and different values of lattice length $N=16,32,64,128$. The index axis is normalized to 1 . (b) The Lyapunov spectra $\lambda_{j}$ for various values of the nonlinearity index $n$ (from bottom to top: $n=1.5,2,2.5,3,3.5,4,6,8)$ and fixed lattice length $N=16$. Larger values of $n$ produce stronger chaos that smaller ones. (c) The largest Lyapunov exponent $\lambda_{1}$ for different values of $n$. The horizontal axis is logarithmic, thus one can see that roughly $\lambda_{0} \sim \operatorname{const} \cdot \log (n)$.

sideration, chaos is observed for arbitrary small energies - only the Lyapunov exponents decrease accordingly.

\section{CONCLUSION}

In this paper we have studied strongly nonlinear Hamiltonian lattices, with a focus on compact traveling waves and on chaos. We have presented an accurate numerical scheme allowing one to find solitary waves. Moreover, from the integral form representation used one easily derives the super-exponential form of the tails. In this way we have confirmed this remarkable result by Chatterjee [5] by another analytical method and by accurate numerical analysis. The constructed compactons were then studied via direct numerical simulations of the lattice. Their collisions are nearly elastic, but the small non-elastic components on a long time scale destroy the localized waves and result in a chaotic state. Chaos appears to be a general statistically stationary state in finite lattices, with a spectrum of Lyapunov exponents where the largest one grows roughly proportional to the logarithm of the nonlinearity index.

We would like to mention here also several aspects that deserve further investigations. Recently, a problem of heat transport in one-dimensional lattices have attracted a large attention [33], here the properties of strongly nonlinear lattices may differ from those possessing linear waves. Also a quantization of these lattices seems to be a non-trivial task, as 
there are no linear phonons to start with. Finally, the Anderson localization property of disordered lattices has been recently intensively discussed for nonlinear systems. For strongly nonlinear lattices the problem has to be attacked separately, as here one cannot rely on the spectral properties of a linear disordered system.

\section{Acknowledgments}

We thank P. Rosenau and D. Shepelyansky for constant stimulating discussions. The work was supported by DFG via Grant PI-220/10 and via Collaborative Research Project 555 "Complex nonlinear processes".

[1] A focus issue on "The "Fermi-Pasta-Ulam" problem - the first 50 years" (ed. by D. K. Campbell, P. Rosenau and G. Zaslavsky), CHAOS 15 (2005).

[2] S. Flach and C. R. Willis, Physics Reports 295, 181 (1998).

[3] V. F. Nesterenko, J. Appl. Mech. Tech. Phys. 5, 733 (1983).

[4] C. Coste, E. Falcon, and S. Fauve, Phys. Rev. E 56, 6104 (1997).

[5] A. Chatterjee, Phys. Rev. E 59, 5912 (1999).

[6] M. A. Porter, C. Daraio, E. B. Herbold, I. Szelengowicz, and P. G. Kevrekidis, Phys. Rev. E 77, 015601(R) (pages 4) (2008).

[7] A. N. Lazaridi and V. F. Nesterenko, J. Appl. Mech. Tech. Phys. 26, 405 (1985).

[8] S. L. Gavrilyuk and V. F. Nesterenko, J. Appl. Mech. Tech. Phys. 34, 784 (1994).

[9] V. Nesterenko, Dynamics of Heterogeneous Materials (Springer, New York, 2001).

[10] P. Rosenau and J. M. Hyman, Phys. Rev. Lett. 70, 564 (1993).

[11] P. Rosenau, Phys. Rev. Lett. 73, 1737 (1994).

[12] R. S. Sinkovits and S. Sen, Phys. Rev. Lett. 74, 2686 (1995).

[13] S. Sen and R. S. Sinkovits, Phys. Rev. E 54, 6857 (1996).

[14] S. Sen, M. Manciu, and J. D. Wright, Phys. Rev. E 57, 2386 (1998).

[15] M. Manciu, V. N. Tehan, and S. Sen, Chaos 10, 658 (2000).

[16] M. Manciu, S. Sen, and A. J. Hurd, Phys. Rev. E 63, 016614 (2000).

[17] S. Sen and M. Manciu, Phys. Rev. E 64, 056605 (2001). 
[18] E. Hascoët and E. J. Hinch, Phys. Rev. E 66, 011307 (2002).

[19] A. Rosas and K. Lindenberg, Phys. Rev. E 68, 041304 (2003).

[20] A. Rosas and K. Lindenberg, Phys. Rev. E 69, 037601 (2004).

[21] S. Job, F. Melo, A. Sokolow, and S. Sen, Phys. Rev. Lett. 94, 178002 (2005).

[22] S. Sen, J. Hong, J. Bang, E. Avalos, and R. Doney, Physics Reports 462, 21 (2008).

[23] G. Friesecke and J. Wattis, Commun. Math. Phys. 161, 391 (1994).

[24] R. S. MacKay, Phys. Lett. A 251, 191 (1999).

[25] P. Rosenau, Phys. Lett. A 311, 39 (2003).

[26] Nesterenko, V. F., Le Journal de Physique IV 04, C8 (1994), URL http://dx.doi.org/doi/10.1051/jp4:19948112.

[27] D. Treschev, Discrete and Continuous Dynamical Systems 11, 867 (2004).

[28] V. I. Petviashvili, Sov. J. Plasma Phys. 2, 257 (1976).

[29] V. I. Petviashvili, Physica D 3, 329 (1981).

[30] M. Abramowitz and I. A. Stegun, Handbook of Mathematical Functions (Department of Commerce USA, Washington, D.C., 1964).

[31] K. Ahnert and M. Abel, Computer Physics Communications 177, 764 (2007).

[32] S. Sen, K. Mohan, and J. Pfannes, Physica A 342, 336 (2004).

[33] S. Lepri, R. Livi, and A. Politi, Physics Reports 377, 1 (2003). 